\title{
Worldwide ACE (I/D) polymorphism may affect COVID-19 recovery rate: an ecological meta-regression
}

\author{
Naser Hatami ${ }^{1}$ - Salma Ahi $^{2}$ - Alireza Sadeghinikoo ${ }^{3} \cdot$ Mahdi Foroughian $^{4} \cdot$ Farshid Javdani $^{1} \cdot$ Navid Kalani $^{5}$. \\ Mostafa Fereydoni ${ }^{1} \cdot$ Pouyan Keshavarz ${ }^{1} \cdot$ Ava hosseini $^{1}$
}

Received: 19 April 2020 / Accepted: 1 June 2020 / Published online: 15 June 2020

(c) Springer Science+Business Media, LLC, part of Springer Nature 2020

\begin{abstract}
With the emergence of the Novel Coronavirus (2019-nCoV), researchers worldwide have started detecting the probable pathogenesis of the disease. The renin-angiotensin system (RAS) and angiotensin-converting enzymes have received a good deal of attention as possible pathways involved in $2019-\mathrm{nCoV}$ pathogenesis. As the experiments seeking to find potential medications acting on these pathways are being conducted in the early phases, having an ecological worldview on the relationship between the prevalence of COVID-19 disease and the genetic differences in the genes involved in the RAS system could be valuable for the field. In this regard, we conducted a meta-analysis study of the prevalence of ACE (I/D) genotype in countries most affected by the COVID-19. In the meta-analysis, 48,758 healthy subjects from 30 different countries were evaluated in 116 studies, using the Comprehensive Meta-analysis software. The I/D allele frequency ratio was pooled by a random-effect model. The COVID-19 prevalence data of death and recovery rates were evaluated as the latitudes for the meta-regression analysis. Our results demonstrated that with the increase of the I/D allele frequency ratio, the recovery rate significantly increased (point estimate: 0.48 , CI 95\%: $0.05-0.91, p=0.027$ ). However, there was no significant difference in the case of death rate (point estimate: 1.74, CI 95\%: 4.5-1.04, $p=0.22$ ). This ecological perspective coupled with many limitations does not provide a direct clinical relevance between the COVID-19 and RAS system, but it shows potential pathophysiological associations. Our results raise concerns about ethnic and genetic differences that could affect the effectiveness of the currently investigated RAS-associated medications in different regions.
\end{abstract}

Supplementary information The online version of this article (https:// doi.org/10.1007/s12020-020-02381-7) contains supplementary material, which is available to authorized users.

Salma Ahi

Salmaahi.61@gmail.com

1 Student Research Committee, Jahrom University of Medical Sciences, Jahrom, Iran

2 Research Center for Noncommunicable Diseases, Faculty of Medicine, Jahrom University of Medical Sciences, Jahrom, Iran

3 Sports Medicine Center, Oxygen Sports Group, Tehran, Iran

4 Department of emergency medicine, Faculty of Medicine, Mashhad University of medical sciences, Mashhad, Iran

5 Research center for social Determinants of Health, Jahrom University of Medical Sciences, Jahrom, Iran

\section{Introduction}

Human coronaviruses (HCoVs) were first identified in the 1960s. Many HCoVs have been identified since then, including those that cause severe acute respiratory syndrome (SARS) and the Middle East respiratory syndrome [1], two conditions that cause fatal respiratory diseases in humans after infection. Coronaviruses (Coves) of the Coronaviridae family, are enveloped viruses with a single-strand, positivesense RNA genome, having the largest known RNA virus genome $[1,2]$. In humans, $\mathrm{CoV}$ infections mainly involve the upper respiratory and gastrointestinal tracts, causing various symptoms ranging from a mild self-limited disease such as common cold to the more severe manifestations such as bronchitis and pneumonia [3]. In 1965, the first human coronavirus (HCV) was isolated from the nasal discharge of common cold patients [4]. Infected patients with the novel coronavirus 2019 (COVID-19) demonstrated clinical manifestations with extreme pneumonia and increased mortality rates. Currently, there is no definite and/ 
or effective treatment for COVID-19 [5]. Sun et al. in their latest publication expressed the possible interaction of COVID-19 and ACE2 which may result in the degradation of ACE2, and the blockage of the pathway to the receptor by ACE2/Ang (1-7)/Mas receptor [6]. The angiotensinconverting enzyme inhibitors have been proposed as probable beneficial treatments for COVID-19, insinuating the RAS system as an important target for the treatment of lung diseases [5].

Comparing the occurrence of the disease in different geographical areas having genetically diverse ethnicities, can provide a scientific foundation for future researches to implement protocols on COVID-19 treatment, considering the quick advent of the coronavirus disease and the current data shortfall on the virus pathophysiology.

\section{Methods}

\section{COVID-19 data}

The event rate for the number of positive cases of coronavirus and its associated death rates for each country was extracted from the WHO website. The inclusion criteria for the countries were as follows: (a) at least 14 days incubation time-lapse from first detected COVID-19 case in the country, (b) more than 1000 detected cases of COVID-19, and (c) availability of studies reporting I/D polymorphism in the country.

\section{ACE polymorphism data}

To evaluate the coronavirus prevalence based on its association with the renin-angiotensin system, studies reporting the prevalence of ACE I/D polymorphism in each country were collected to estimate the pooled I/D ratio, considering the Systematic Studies and Meta-Analysis Reporting System (PRISMA) statement. To select the studies reporting ACE I/D polymorphism prevalence, ISI, PubMed, Scopus, and Google Scholar databases were searched with keywords such as ACE gene, Polymorphism, I/D, and the name of each country from the year 1990 to 2020 . Three researchers simultaneously searched for the published articles. If previous meta-analyses were available in any particular country, the same study was referred to. In the first step, 1399 studies were evaluated based on their titles and after excluding 84 duplicated studies, 154 studies were also excluded due to their irrelevance. In the full-text review of the selected articles, 431 were excluded as a result of the study having been conducted either in a false study region, or not having healthy controls, or not reporting I/D polymorphism. The main inclusion criterion was, providing the general prevalence of ACE I/D polymorphism in the healthy population. Finally, 116 articles were used for data extraction. The dataset of I and D allele frequency in the healthy control group of each study was extracted. Data were analyzed based on the random-effects model using the Comprehensive Meta-Analysis software version 2. $I^{2}$ indices were used to determine the heterogeneity between studies. As the $I^{2}$ index of pooled I/D allele frequency ratio was $99 \%$, random-effects models were used. The inverse method was also applied to estimate the variance and weight for each study. The bias of publication was assessed by Egger's regression intercept.

\section{Results}

To assess the pooled I/D allele frequency (Figs. 1 and 2) ratio in each country, 116 studies (reporting the (I/D) polymorphism genotypes in healthy controls) were included in the study. The last update of the 2nd April 2020 statistics of the World Health Organization was extracted based on each country's total positive cases, total deaths, and total recovered patients.

Meta-regression of moderator variables of the total death rate and the total recovered rate vs the point estimate of the I/D allele frequency ratio were provided as shown in Table 1. The dataset of all included studies are available in supplementary Table S1.

The results of the meta-analysis of the pooled I/D allele frequency ratio of included studies are shown in Table 1. The point estimate of ratio was 0.403 (CI 95\%: 0.398-0.409). In all countries, the I/D allele frequency ratio was below 1 except in South-Africa, China, Japan, Israel, and Denmark, where the value was above 1 . The average I/D allele ratio in Europe was 0.55 , while it was 0.93 in the whole of Asia. No evidence of bias in the publication has been found in the overall analysis (Egger's $p$ value $=0.281)$.

The results of the meta-regression are shown in Figs. 2 and 3. With the increase of the I/D allele ratio in the studied countries, the recovery rate significantly increased (point estimate: 0.48, CI 95\%: 0.05-0.91, $p=0.027$ ). Meanwhile, there was no significant difference in the case of death rate (point estimate: -1.74 , CI 95\%: -4.5 to $1.04, p=0.220$ ).

\section{Discussion}

Our current knowledge of the new COVID-19 is rapidly being augmented. Researchers around the world are looking forward to understanding the pathogenesis of the disease.

Our study revealed a possible relationship between ACE I/D allele frequency and the COVID-19 recovery rate. Also, this study showed that the ACE I/D allele ratio is very 


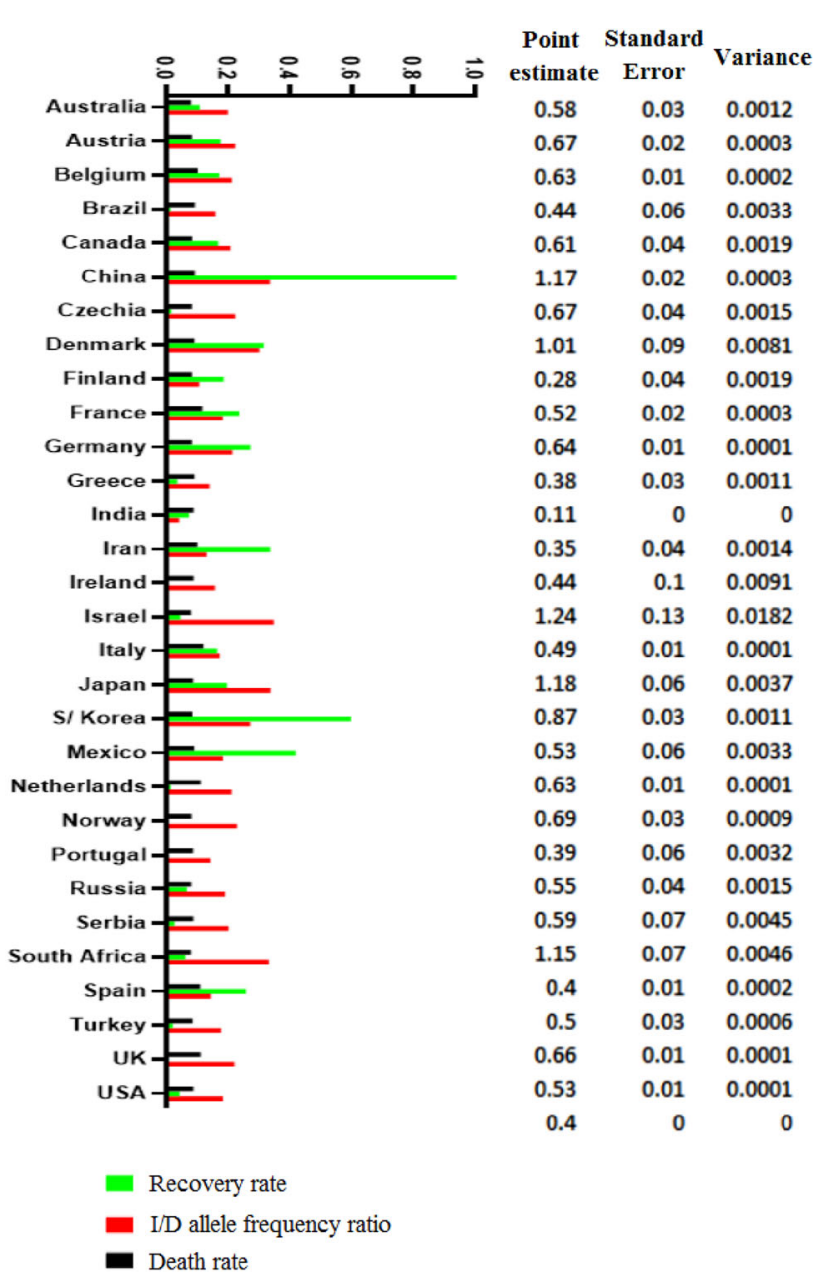

Fig. 1 The Forrest plot of the pooled analysis of I/D polymorphism ratio. Columns on left are log-transferred values of I/D allele frequency

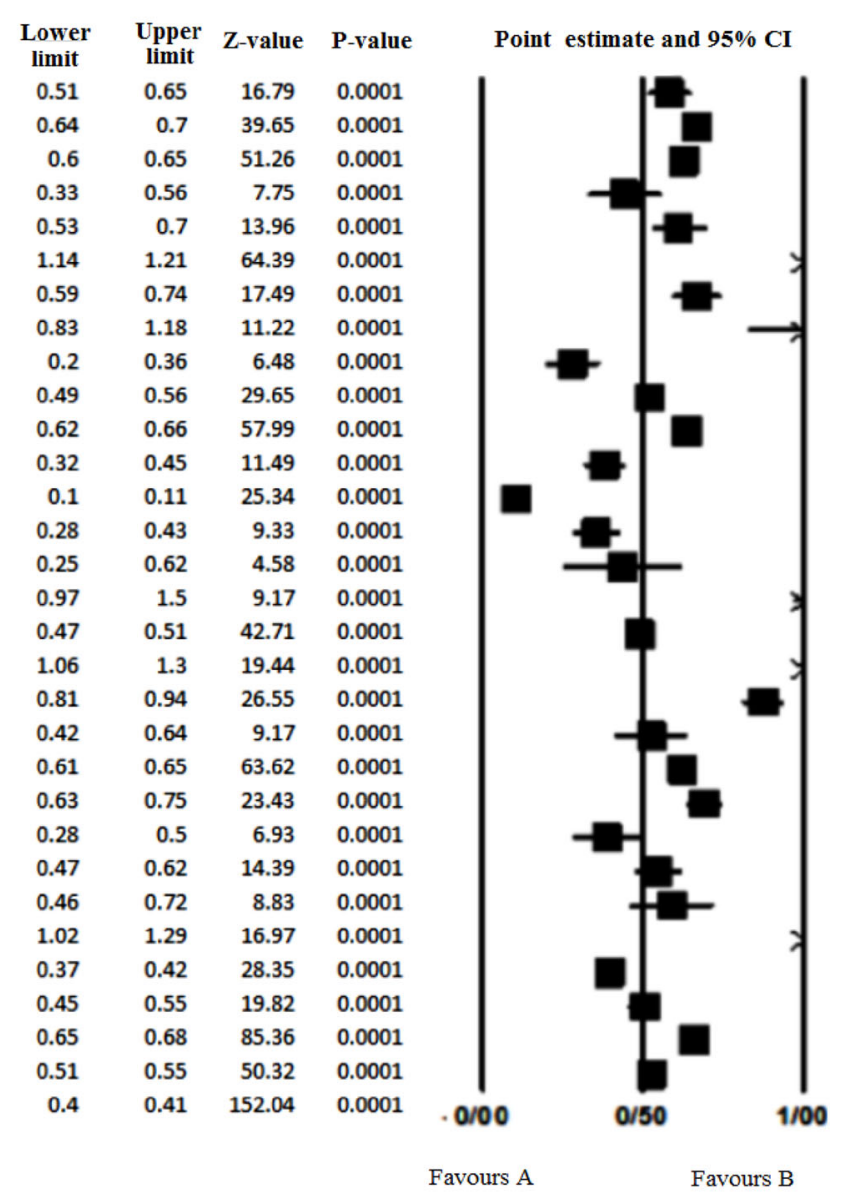

ratio, recovery, and death rate. The point estimate of the I/D allele frequency ratio is shown at the right side in the forest plot
Fig. 2 Meta-regression of I/D allele frequency ratio vs. recovery rate

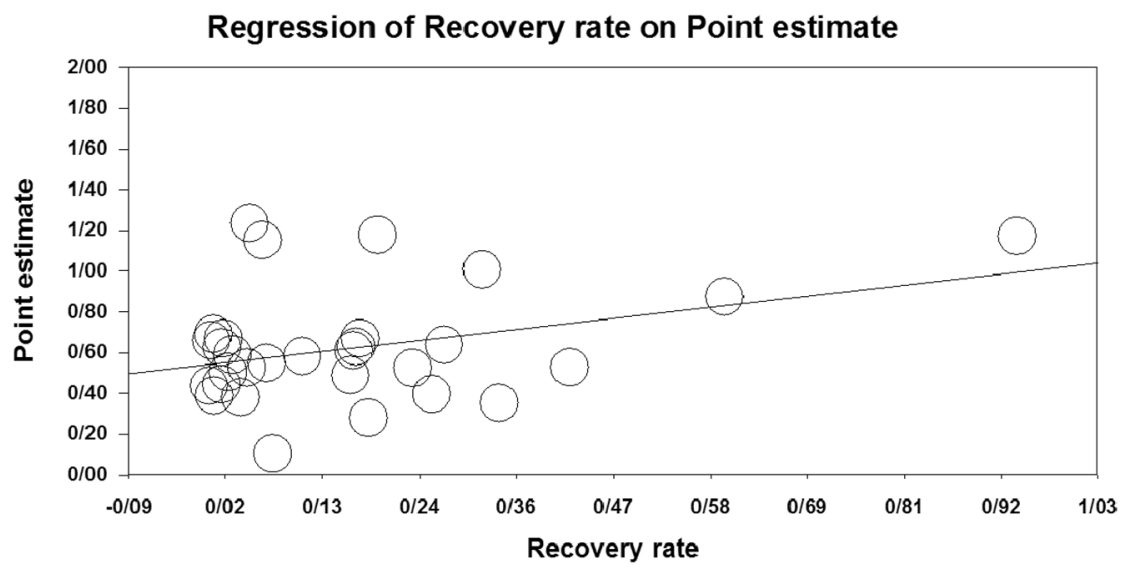

diverse worldwide. East Asian countries such as China and Japan all had a ratio of more than 1, showing a higher rate of I allele frequency. In East Asia, there appeared to be more I-alleles than $\mathrm{D}$ in the population genome of the people. However, South Korea has an allele ratio of 0.87 , which is still much higher than in European countries. These findings are consistent with studies of ACE I/D genetic mapping.

Similar to our report, Saab et al. examined the average ACE II genotype frequency in healthy subjects in different 
Table 1 Included countries characteristics

\begin{tabular}{|c|c|c|c|c|c|c|c|}
\hline & $\mathrm{DD}$ & $\mathrm{I} / \mathrm{D}$ & II & $\mathrm{D}$ allele frequency & I allele frequency & Death ${ }^{a}$ & Recovery $\%^{\circ}$ \\
\hline Australia & 134 & 216 & 94 & 484 & 282 & 0.52 & 10.93 \\
\hline Austria & 619 & 1108 & 524 & 2346 & 1572 & 1.46 & 17.6 \\
\hline Belgium & 1074 & 2030 & 876 & 4178 & 2628 & 6.82 & 17.13 \\
\hline Brazil & 51 & 33 & 20 & 135 & 60 & 4.17 & 1.54 \\
\hline Canada & 85 & 149 & 65 & 319 & 195 & 1.47 & 16.85 \\
\hline China & 1154 & 1228 & 1382 & 3536 & 4146 & 4.07 & 93.81 \\
\hline Czech & 116 & 225 & 102 & 457 & 306 & 1.3 & 1.76 \\
\hline Denmark & 41 & 43 & 42 & 125 & 126 & 3.7 & 31.75 \\
\hline Finland & 49 & 52 & 14 & 150 & 42 & 1.24 & 18.58 \\
\hline France & 470 & 736 & 293 & 1676 & 879 & 11.01 & 23.7 \\
\hline Germany & 1345 & 2581 & 1121 & 5271 & 3363 & 1.37 & 27.35 \\
\hline Greece & 106 & 134 & 44 & 346 & 132 & 3.66 & 3.78 \\
\hline India & 130 & 316 & 214 & 6108 & 642 & 2.8 & 7.48 \\
\hline Iran & 75 & 96 & 29 & 246 & 87 & 6.19 & 33.72 \\
\hline Ireland & 12 & 24 & 7 & 48 & 21 & 2.81 & 0.12 \\
\hline Israel & 9 & 50 & 28 & 68 & 84 & 0.57 & 4.81 \\
\hline Italy & 1185 & 1358 & 608 & 3728 & 1824 & 12.25 & 16.49 \\
\hline Japan & 68 & 185 & 126 & 321 & 378 & 2.41 & 19.64 \\
\hline Korea & 256 & 294 & 235 & 806 & 705 & 1.73 & 59.84 \\
\hline Mexico & 40 & 79 & 28 & 159 & 84 & 3.31 & 41.92 \\
\hline Netherlands & 1705 & 3056 & 1349 & 6466 & 4047 & 9.46 & 1.59 \\
\hline Norway & 208 & 378 & 183 & 794 & 549 & 1.08 & 0.6 \\
\hline port & 39 & 45 & 16 & 123 & 48 & 2.49 & 0.69 \\
\hline Russia & 126 & 125 & 69 & 377 & 207 & 0.82 & 6.77 \\
\hline Serbia & 30 & 72 & 26 & 132 & 78 & 2.64 & 2.85 \\
\hline South-Africa & 46 & 158 & 96 & 250 & 288 & 0.6 & 6.31 \\
\hline Spain & 630 & 767 & 268 & 2027 & 804 & 9.29 & 25.92 \\
\hline Turkey & 238 & 303 & 131 & 779 & 393 & 2.03 & 2.31 \\
\hline UK & 2896 & 5241 & 2429 & 11033 & 7287 & 9.45 & 0.35 \\
\hline USA & 1331 & 2145 & 844 & 4807 & 2532 & 2.56 & 4.51 \\
\hline
\end{tabular}

a Based on WHO situation dashboard, available at https://www.who.int/redirect-pages/page/novel-corona virus-(COVID-19)-situation-dashboard and World Meter webpage (https://www.worldometers.info/corona virus/) on 2 April 2020
Fig. 3 Meta-regression of I/D allele frequency ratio vs. death rate

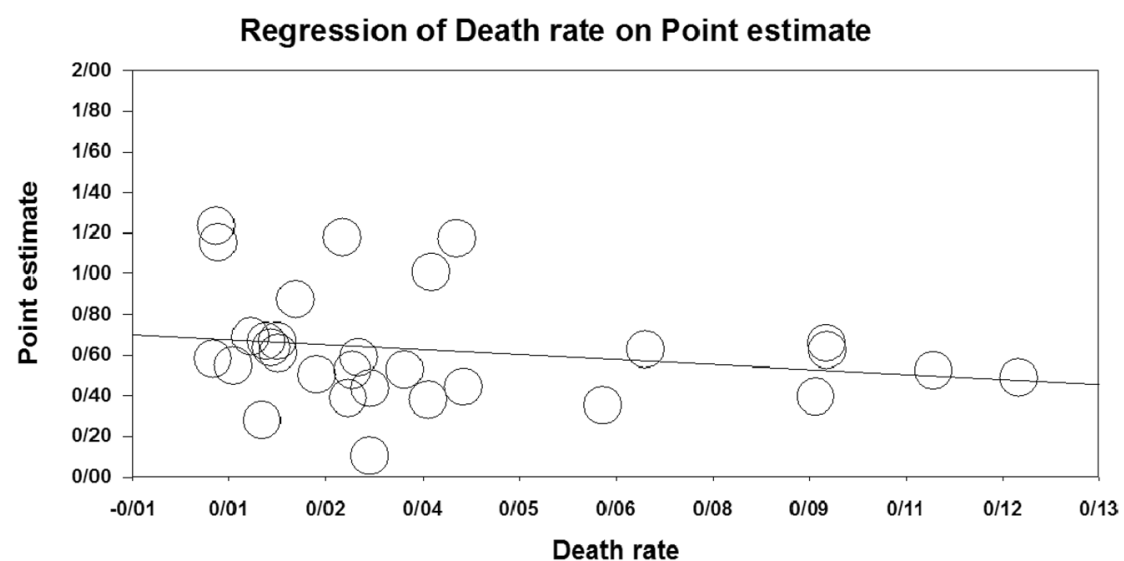

populations of different countries [6]. They disclosed that ACE I allele and genotype frequencies show an association with longitude. A regional analysis perpetrated a strong correlation between II genotype frequency and longitude, indicated by a decrease in the II frequency from Europe toward the Middle East, and an alternate east-bound rise toward Asia. Summarily, the average frequency of the II genotype in the north of Europe and Denmark was $23 \%$, while the UK and Spain recorded $20 \%$ and $15 \%$, whereas northern and southern Italy registered $14 \%$ and $12 \%$, with 
China and Japan averaging $35 \%$ and $45 \%$ respectively [6]. Also, this finding was confirmed in our study, as Denmark had an ACE I/D ratio of 1.01, which was higher than in other European countries. The interesting finding of Saab et al.'s study about the lowest II genotypes of Europe in Italy, makes us think about a possible role of genetic factors and the tragedy of the COVID-19 outbreak in Italy with a high case-fatality rate [7]. European countries like Italy, Spain, and the UK have been widely affected by COVID19. It is well known that ACE I allele frequency in Europe is lower than in East Asia as seen in our study, which indicated the average ACE I/D allele ratio in Europe as 0.55 , while it was 0.93 in the whole of Asia. This finding confirms the results of Wang et al.'s study as they estimated the average ACE insertion frequency in East Asia to be 0.63 and 0.43 in Europe [8].

The diversity in the mode of disease dissemination in different regions can be attributed to a large number of genetic and environmental influences, and this has led researchers to investigate the possible interconnection between environmental influences such as weather with the COVID-19 pandemic [9]. Noteworthy is the fact that, whereas the synthetic temperature factor has been positively associated with D allele, synthetic humidity factor, and D allele are negatively linked in terms of global regional spread, and interdependence between these two factors and the D allele assumes an out of Africa expansion pattern in space [10]. Per our results regarding the ACE I/D ratio, Li et al. had shown that the highest frequency of $\mathrm{D}$ allele appears in Africa, Europe, America, and the Arab regions, whereas the lowest frequency is located in East Asia. Based on their study USA is located in a relative high frequency of D allele [10] which was confirmed by our study and this high $\mathrm{D}$ allele frequency may be associated with bad outcomes of COVID-19 in this country.

Researchers have suggested that the virus binds to the ACE2 receptor on the surface of human cells through its envelope spike glycoprotein and enters the cell [11]. On the other hand, in the study of Monteil et al. it was found that human recombinant soluble ACE2 could block SARS-CoV2 cell attachment [12]. A comprehensive review by Fan et al. underscored the close relationship between ACE2 and ACE through which ACE2 regulates RAS, exhibiting a greater than $40 \%$ identity with the somatic ACE catalytic domain [13]. Still in the perspective of ACE, Cao et al. evaluated the allele frequency of 62 variants in the coding regions of ACE2 in different populations. Despite demonstrating the predisposition of the ACE2 gene polymorphism in different populations worldwide, they, however, could not pinpoint any specific evidence to confirm the presence of the ACE2 binding-resistant coronavirus S-protein mutants in various populations [14]. Also, not all lung tissue cells expressed ACE2 and its expression was concentrated in some type II alveolar cells [15]. Cao et al. found specific polymorphisms associated with higher ACE2 expression in the East Asian population [14]. In our study, the highest I/D allele ratio was seen in China and Japan, in East Asia. This similarity reinforces the idea of the population's difference in the RAS system that may predispose them to a divergent susceptibility to the disease.

While the genetic predisposition of the ACE genes in COVID-19 patients has not yet been examined, the relationship between other respiratory diseases and the ACE genotype has been investigated in various studies. In one study that investigated the relationship between the ACE genotype and the occurrence of acute respiratory distress syndrome, it was found that the frequency of DD genotype of patients was significantly higher than that of healthy controls [16]. This conforms with our finding which demonstrates that, with the increase of the $\mathrm{I} / \mathrm{D}$ allele ratio (I allele increases or D allele decreases), the recovery rate increases. Hence, it is not surprising to see a higher recovery rate in East Asian countries such as Japan (point estimate: 1.18 , CI 95\%: 1.06 to 1.3 ), China (point estimate: 1.17, CI 95\%: 1.14 to 1.21 ), and S. Korea (point estimate: 0.87 , CI 95\%: 0.81-0.94), $P=0.0001$, due to the high I/D allele ratio in the population. As expected, the worst-hit European countries like Spain (point estimate: 0.4, CI 95\%: 0.37-0.42), Italy (point estimate: 0.49 , CI 95\%: 0.47-0.51), and UK (point estimate: 0.66 , CI 95\%: 0.65 to 0.68 ), $P=$ 0.0001 , all have low I/D allele ratio from the Forest Plot analysis. Also in another study, the researchers found that the D allele had a role in the progression of noninfectious pulmonary dysfunction [17].

That notwithstanding, this study is restricted by the varied known and unknown factors affecting the global recovery and death rate of COVID-19 patients, including demographic characteristics such as patient's sex, age, underlying diseases, and the various approaches used to treat patients in the different regions. These data were not yet publicly available worldwide to be considered in our study. Besides, the utilization of official numbers of COVID-19 infected people are biased by the number of correct diagnoses that are carried out in each country, and this might consequently affect the recovery and death rates.

\section{Conclusion}

Despite the limitations cited above, our result demonstrating the positive correlation of the I/D ACE gene allele ratio with the recovery rate of Covid-19 patients presents a hypothesis worth considering for further studies. We suggest case-control studies to assess the role of the definitive ACE gene polymorphism on treatment outcomes. The 
importance of our finding is rooted in the possibility that these genetic variations may cause different responses to the COVID-19 treatments focusing on the RAS system components, which are currently being investigated in experimental studies.

Author contributions The manuscript has been read and approved by all authors. S.A. and N.H. designed the study. S.A and N.H undertook data analysis. F.J., P.K., M.F., A.H., M.fe, and A.S. conducted the searches and collected the data. A.H., F.J., P.K., N.K., N.H., A.S., and M.F. wrote the draft manuscript. S.A., A.H., M.F and N.H. revised the manuscript.

\section{Compliance with ethical standards}

Conflict of interest The authors declare that they have no conflict of interest.

Publisher's note Springer Nature remains neutral with regard to jurisdictional claims in published maps and institutional affiliations.

\section{References}

1. S.R. Weiss, S. Navas-Martin, Coronavirus pathogenesis and the emerging pathogen severe acute respiratory syndrome coronavirus. Microbiol. Mol. Biol. Rev. 69(4), 635-664 (2005)

2. Lai. M. C, Coronaviridae. Fields virology 1, 1305-1318 (2007)

3. B.A. Wevers, L. van der Hoek, Recently discovered human coronaviruses. Clin. Lab. Med. 29(4), 715-724 (2009)

4. D.A. Tyrrell, M.L. Bynoe, Cultivation of a novel type of commoncold virus in organ cultures. Br. Med. J. 1, 1467-1470 (1965)

5. M.L. Sun, J.M. Yang, Y.P. Sun, G.H. Su, Inhibitors of RAS Might Be a Good Choice for the therapy of COVID-19 Pneumonia. Chin. J. Tuberc. Respir. Dis. 43(Feb), E014 (2020)

6. Y.B. Saab, P.R. Gard, A.D. Overall, The geographic distribution of the ACE II genotype: a novel finding. Genet. Res. 89(4), 259-267 (2007)

7. E. Livingston, K. Bucher, Coronavirus disease 2019 (COVID-19) in Italy. JAMA. 323(14), 1335 (2020)
8. K. Wang, Y. Li, C. Dai, K. Wang, J. Yu, Y. Tan, W. Zhang, X.F. $\mathrm{Yu}$, Characterization of the relationship between APOBEC3B deletion and ACE Alu insertion. PloS one. 8(5), e64809 (2013)

9. R. Tosepu, J. Gunawan, D.S. Effendy, L. Ode, A. Imran Ahmad, H. Lestari, H. Bahar, P. Asfian, Correlation between weather and Covid-19 pandemic in Jakarta, Indonesia. Sci. Total Environ. 725, 138436 (2020). https://doi.org/10.1016/j.scitotenv.2020.138436

10. X. Li, X. Sun, L. Jin, F. Xue, Worldwide spatial genetic structure of angiotensin-converting enzyme gene: a new evolutionary ecological evidence for the thrifty genotype hypothesis. Eur. J. Hum. Genet. 19(9), 1002-1008 (2011)

11. F. Wu, S. Zhao, B. Yu, Y.M. Chen, W. Wang, Z.G. Song, Y. Hu, Z.W. Tao, J.H. Tian, Y.Y. Pei, M.L. Yuan, A new coronavirus associated with human respiratory disease in China. Nature. 579 (7798), 265-269 (2020)

12. Monteil et al., Inhibition of SARS-CoV-2 infections in engineered human tissues using clinical-grade soluble human ACE2. cell press pre-proof. https://doi.org/10.1016/j.cell.2020.04.004

13. X.H. Fan, Y.B. Wang, H. Wang, K. Sun, W.L. Zhang, X.D. Song, J.Z. Cheng, H.Y. Wu, X.L. Zhou, R.T. Hui, Polymorphisms of angiotensin-converting enzyme (ACE) and ACE2 are not associated with orthostatic blood pressure dysregulation in hypertensive patients. Acta Pharmacol. Sinica 30(9), 1237-1244 (2009)

14. Y. Cao, L. Li, Z. Feng, S. Wan, P. Huang, X. Sun, F. Wen, X. Huang, G. Ning, W. Wang, Comparative genetic analysis of the novel coronavirus (2019-nCoV/SARS-CoV-2) receptor ACE2 in different populations. Cell Discov. 6(1), 1-4 (2020)

15. Y. Zhao, Z. Zhao, Y. Wang, Y. Zhou, Y. Ma, W. Zuo, Single-cell RNA expression profiling of ACE2, the putative receptor of Wuhan 2019-nCov. BioRxiv. (2020). https://doi.org/10.1101/ 2020.01.26.919985

16. R.P. Marshall, S. Webb, G.J. Bellingan, H.E. Montgomery, B. Chaudhari, R.J. McAnulty, S.E. Humphries, M.R. Hill, G.J. Laurent, Angiotensin converting enzyme insertion/deletion polymorphism is associated with susceptibility and outcome in acute respiratory distress syndrome. Am. J Respir. Crit. Care Med. 166 (5), 646-650 (2002)

17. M. Onizuka, M. Kasai, T. Oba, Y. Atsuta, S. Terakura, R. Suzuki, K. Kitaori, K. Miyamura, T. Hotta, Y. Kodera, Increased frequency of the angiotensin-converting enzyme gene D-allele is associated with noninfectious pulmonary dysfunction following allogeneic stem cell transplant. Bone Marrow Transplant. 36(7), 617-620 (2005) 